Pacific Journal of Mathematics

STRONG CARLEMAN AND STRONG UNIFORM 


\title{
STRONG CARLEMAN AND STRONG UNIFORM APPROXIMATION
}

\author{
ANNFTte SinclaIR
}

\begin{abstract}
A primary objective of this article is to establish and apply relations between Carleman approximation and strong Carleman approximation, also between uniform and strong uniform approximation. Strong Carleman and strong uniform approximation, as herein defined, imply Carleman and uniform approximation, respectively.
\end{abstract}

Results are given for approximation on a closed set $E$ on an open Riemann surface $\Re$, hence include, in particular, approximation on closed subsets of the complex plane and of the real axis. If every function $f \in A(E)$ (that is, $f$ continuous on $E$ and analytic on the interior $E^{\circ}$ of $E$ ) can be Carleman (alternatively, uniformly) approximated on $E$ by functions of $M(\Re)$ (that is, by functions meromorphic on $\Re$ ), then every such function $f$ can also be strongly-Carleman (strongly-uniformly) approximated on $E$ by meromorphic functions. (See Theorem 2 and Corollary 2.1.)

For both strong Carleman and strong uniform approximation, the closeness of approximation required is stipulated by a continuous function $\varepsilon=\varepsilon(p)$. With appropriate restrictions in a neighborhood of each of its zeros, the function $\varepsilon$ is allowed zeros on $E^{\circ}$ and at some points of $\partial E$, whereas for Carleman approximation ([6], [7], [10]), the continuous function $\varepsilon=\varepsilon(p)$ specifying closeness of approximation is bounded away from zero on every compact subset of $E$. Whether $\varepsilon$ is allowed to vanish at a particular boundary point of $E$ depends on the behavior of $f$ near that point. When $f$ is the function to be approximated, an interesting possibility as an $\varepsilon$-function is $r f(p)$, where $r$ is a real number between 0 and 1 , although this is not always an admissible $\varepsilon$-function.

The function $f$ to be approximated on $E$ is meromorphic on $E^{\circ}$ and continuous on $E$, except at poles, and may even have 'poles' of a restricted type on $\partial E$. (We shall abbreviate by writing: $f \in M(E)$.) An assigned inequality $|f(p)-g(p)| \leq|\varepsilon(p)|$, of course, requires that $g$ interpolates $f$ at each zero of $\varepsilon=\varepsilon(p)$, except that if $f$ has a pole at such a zero $q \in E$, then $\lim _{p \rightarrow q, p \in E}(f(p)-g(p))=0$. 
For strong Carleman approximation, the $\varepsilon$-closeness of approximation is assigned by an $\varepsilon_{E}$-function. A function $\varepsilon=\varepsilon(p)$ which is continuous on the closed set $E$ will be called an $\varepsilon_{E}$-function if, for each zero $q$ of $\varepsilon$ on $E$, there is a smallest positive integer $n_{q}$, called the order of $q$, and a neighborhood $\mathscr{N}_{q}$ such that the following condition holds: the function defined as $\varepsilon(p) /(p-q)^{n_{q}}$ is nonvanishing and continuous on $\left(\mathscr{N}_{q} \cap E\right)$, except for a removable singularity at $q$. Corresponding to given $f$ continuous on $E$ (except for poles), we shall say that an $\varepsilon_{E}$-function is $f$-admissible provided that, for each zero $q$ of $\varepsilon$ (order $n_{q}$ ), there is a neighborhood $\mathscr{N}_{q}$ of $q$ such that $f(p) /(p-q)^{n_{q}} \in M\left(\mathscr{N}_{q} \cap E\right)$.

With $A(T)$ indicating the set of functions continuous on $T(\subset \Re)$ and analytic on $T^{\circ}$, the interior of $T, A(\Re)$ denotes the set of functions analytic on $\Re$. We let $A(\Re)_{O E}$ designate the subset of $A(\Re)$ whose elements are nonvanishing in $\Re \backslash E$.

For strong uniform approximation, an $\varepsilon_{E, \infty}$-function is used. We shall call a function $\varepsilon$, which is continuous on $E$, an $\varepsilon_{E, \infty}$-function if there exists $h \in A(\Re)_{O E}$ such that $\varepsilon(p) / h(p)$ is bounded from zero when $p \in E$. If we can also require that $f / h \in M(E)$, then we shall call $\varepsilon$ an $f$-admissible $\varepsilon_{E, \infty}$-function.

If, for given $f$ defined on $E$ and an arbitrary $f$-admissible $\varepsilon_{E}$-function $\varepsilon=\varepsilon(p)$, there exists $g$ in a specified class $M$ such that $|f(p)-g(p)| \leq$ $|\varepsilon(p)|$ when $p \in E$, we shall say that $f$ can be strongly-Carleman approximated on $E$ - for brevity, str.-C. approximated on $E$ - by functions of $M$. If, for an arbitrary $f$-admissible $\varepsilon_{E, \infty}$-function $\varepsilon=\varepsilon(p)$, there exists $g \in M$ such that $|f(p)-g(p)| \leq|\varepsilon(p)|$ when $p \in E$, we shall say that $f$ can be strongly-uniformly (str.-u.) approximated on $E$ by functions of $M$.

For a special closed set called a $Q$-set, approximation of a Carleman type, also of a strong Carleman type, by meromorphic functions has been studied by the author in earlier papers $(1949,1952,1958,1965)$. As a special case of an abstract approach to Carleman approximation, with interpolation conditions (1958), existence of a meromorphic approximant to a function of $A(E)$ was shown for the special case that $E$ is the union of disks tangent externally along the real axis. For a closed set $E$ on an open Riemann surface $\Re$, the relation between approximation of functions of $A(E)$ and of $M(E)$, also the relation between Carleman approximation and strong Carleman approximation, was studied. (See [27], also [26].)

Proofs of some main results in this paper rely heavily on a theorem of S. Scheinberg ([23], Theorem 1), which relates approximation of analytic and of meromorphic functions and permits assignment of poles, also upon 
a theorem of Gauthier ([10], Theorem 4), which deals with Carleman approximation on a closed set with empty interior. Solutions of the Cousin Problems 1 and 2 are basic in proofs used here.

For other closely related work, the reader is referred to Arakelyan (1964, 1970); Gauthier (1975, 1977); Kodama (1965); H. J. Landau (1954); Nersesian (1971, 1972); Roth (1938, 1973, 1976); Schienberg $(1976,1978)$.

1. Introduction to some main results. Clearly, strong Carleman approximation of functions of $M(E)$ includes both Carleman and uniform approximation as special cases. Also strong uniform approximation includes uniform approximation. By Lemma $2.1 \S 2$ of this paper), if $f$ can be strongly-Carleman approximated on $E$ then $f$ can be Carleman approximated on $E$, with interpolation conditions pre-assigned on any $f$-admissible interpolation subset of $E$.

An isolated subset $Z$ of $E$ will be called an $f$-admissible interpolation subset of $E$ if, for each point $q \in Z$, there is a neighborhood $\mathscr{N}_{q}$ and a largest positive integer $k_{q}$ such that $\left((f(p)-f(q)) /(p-q)^{k_{q}}\right) \in$ $A\left(\mathscr{N}_{q} \cap E\right)$. The integer $k_{q}$ is called the order of $f$ at $q$.

A function $g$ is said to interpolate $f$ on an $f$-admissible interpolation set $Z$ if, for each point $q(\in Z)$, the function defined by $(f(p)-g(p)) /(p-q)^{k_{q}}$ belongs to $A\left(\mathscr{N}_{q} \cap E\right)$ for some neighborhood $\mathscr{N}_{q}$, where $k_{q}$ is the order of $f$ at $q$.

Suppose $s_{\nu}$ is a point of $E$, which may be a boundary point, and that $\lim _{p \rightarrow s, p \in E^{\nu}}(f(p))=\infty$. Then we shall say that $f$ has a pole at $s_{\nu}$ provided that there exists a neighborhood $\mathscr{N}_{S_{v}}$ of $s_{\nu}$ and a polynomial $P$ in $1 /\left(p-s_{\nu}\right)$, say of degree $n_{\nu}$ such that $f-P$ is analytic on $\left(\mathscr{N}_{S_{\nu}} \cap E\right)^{\circ}$ and is continuous on $\mathscr{N}_{S_{v}} \cap E$.

We have let $A(T)$ designate the set of functions continuous on $T$ $(\subset \Re)$ and analytic on $T^{\circ}$, the interior of $T$. The subset of $A(\Re)$ whose elements are nonvanishing in $\Re \backslash E$ we have denoted by $A(\Re)_{O E}$. If, except possibly for poles, $f$ is analytic on $T^{\circ}$ and continuous on $T$, we write: $f \in M(T)$. Then $M(\Re)$ denotes the set of functions analytic everywhere on $\Re$ except for poles. We let $M_{S}(T)$ be the subset of $M(T)$ whose poles lie on $S(\subset T)$. With $N(T)$ the set of functions, each of which is meromorphic on some open set containing $T$, we let $H(T)$ specify the subset of $N(T)$ consisting of functions each of which is analytic on some open containing $T$. Then $N_{S}(T)$ is the subset of $N(T)$ each element of which has any poles which lie on $T$ at points of $S$. 
TheOREM 1. Suppose the closed set $E$ is contained in $\Re$, where $\Re$ is an open Riemann surface, and that $P \subset \Re \backslash E$. A function $f \in M(E)$ can be strongly-Carleman (alternatively, strongly-uniformly) approximated on $E$ by functions of $M_{E \cup P}(\Re)$ if and only if, for arbitrary $h$ in $A(\Re)_{O E}$ such that $f / h \in M(E)$ the function $f / h$ can be Carleman (alternately, uniformly) approximated on $E$ by functions of $M_{E \cup P}(\Re)$.

THEOREM 2. Suppose $E$ is a closed set on an open Riemann surface $\Re$, and that $P \subset \Re \backslash E$. Then for uniform approximation (a), (b), and (c) are equivalent. For Carleman approximation (a), (b), (c), and (d) are equivalent.

(a) Every function of $A(E)$ can be Carleman (alternately, uniformly) approximated on $E$ by functions of $M_{P}(\Re)$.

(b) Every function of $M(E)$ can be Carleman (alternately, uniformly) approximated on $E$ by functions of $M_{E \cup P}(\Re)$.

(c) Every function of $M(E)$ can be strongly-Carleman (alternately, strongly-uniformly) approximated on $E$ by functions of $M_{E \cup P}(\Re)$.

(d) Every function $f \in M(E)$ can be Carleman approximated on $E$ by functions of $M_{E \cup P}(\Re)$ which interpolate $f$ on any preassigned $f$-admissible interpolation subset of $E$.

Paul M. Gauthier observed that, in (d) of Theorem 2, strong-uniform approximation cannot be substituted as an alternate condition for strong Carleman approximation. For that would yield as a corollary an untrue result, namely: 'If every function of $A(E)$ can be uniformly approximated on $E$ by functions of $M(\Re)$, then an arbitrary function $f \in M(E)$ can be uniformly approximated on $E$ by functions of $M(\Re)$ which interpolate $f$ on any preassigned isolated subset of $E^{\circ}$.' A counterexample to this can be obtained by applying a known theorem (see Titchmarsh, The Theory of Functions, §5.81). "If $f$ is regular and of the form $O\left(e^{k|z|}\right)$, where $k<\pi$ for $\operatorname{Re} z \geq 0$, and $f(z)=0$ for $z=0,1,2, \ldots$, then $f(z)=0$ identically."

COROLlaRY 2.1. If $P$ is not assigned, the theorem remains true, with $M(\Re)$ substituted for both $M_{P}(\Re)$, and $M_{E \cup P}(\Re)$.

Corollary 2.2. (Theorem 2 with $H(E)$ substituted for $A(E)$ and $N(E)$ for $M(E)$.)

Theorem 2 (Parts (a) and (b)) is related to a theorem of Scheinberg published in 1979 ([23], Theorem 1). Under appropriate restrictions on $E$,

\footnotetext{
${ }^{1}$ Theorem 1 and Lemma 2.2 remain true when $N(E)$ is substituted for $M(E)$ and $H(E)$ for $A(E)$.
} 
he showed: Any function which is locally approximable on $E$ by functions of $A(\Re)$ can be uniformly approximated on $E$ by functions of $M_{P}(\Re)$ if and only if any function which is locally approximable on $E$ by functions of $M(\Re)$ is uniformly approximable on $E$ by functions of $M_{E \cup P}(\Re)$.

A function $f$ is said to be locally approximable on $E$ by functions in a class $M$ if each point of $E$ is interior to a compact disk $\bar{D}$ such that $f$ is the uniform limit on $(\bar{D} \cap E)$ of functions of class $M$. For certain closed subsets of the plane (See [25], Theorems 10 and 11), a method used by the author (1952) shows equivalence of Carleman approximation to $f \in H(E)$ and to $f \in N(E)$. The method was generalized ([27], Theorem 1) to show equivalence of Carleman approximation to a function of $A(E)$ by functions of $M_{P}(\Re)$ and of a function of $M(E)$ by functions of $M_{E \cup P}(\Re)$.

A closed subset of $\Re$ is said to be essentially of finite genus if it has a covering by a family of pairwise disjoint open sets, each of which is of finite genus. (See Gauthier [10].)

Next, certain hypotheses for a closed set $E$, containing more than one point, on an open Riemann surface $\Re$ and for $P \subset \Re \backslash E$ are stated for reference: (See $S$. Schienberg [23].) If $\Re$ is the complex plane, then $\left(h_{1}\right)$ is automatically satisfied.

$\left(\mathrm{h}_{1}\right) E$ is essentially of finite genus.

$\left(\mathrm{h}_{2}\right)$ For every compact set $K$ on $\Re$, there is a compact $K^{\prime \prime}$ which contains every bounded component of $\Re \backslash(E \cup K)$ which contains no point of $P$. (For $K=\varnothing$, it is understood that $K^{\prime \prime}=\varnothing$.)

Corollary 2.3. Assume Hypotheses $\left(\mathrm{h}_{1}\right)$ and $\left(\mathrm{h}_{2}\right)$ for $E$ a closed set on an open Riemann surface $\Re$, also that $P \subset \Re \backslash E$. Suppose that every function of $A(E)$ is locally approximable on $E$ by functions of $A(\Re)$. Then every function of $M(E)$ can be strongly-uniformly approximated on $E$ by functions of $M_{E \cup P}(\Re)$.

THEOREM 3. Let $E$ be a closed set on an open Riemann surface $\Re$, and suppose $P \subset \Re \backslash E$. Assume $\left(\mathrm{h}_{1}\right)$ and $\left(\mathrm{h}_{2}\right)$. Then $f \in M(E)$ can be strongly-uniformly approximated on $E$ by functions of $M_{E \cup P}(\Re)$, provided that for any $h$ of $A(\Re)_{O E}$ such that $f / h \in M(E)$, the function $f / h$ is locally approximable on $E$ by functions of $M(\Re)$.

The next theorem is based on Gauthier's analogous theorem ([10], Theorem 4) for Carleman approximation.

THEOREM 4. Suppose $E$ is a closed set, on an open Riemann surface $\Re$, with empty interior. Then an arbitrary function continuous on $E$ (or just in 
$M(E))$ can be strongly Carleman approximated on $E$ by functions of $M(\Re)$ provided that, for arbitrary $f$ continuous on $E$ and for each compact set $K$, the function $f$ can be uniformly approximated on $E \cap K$ by functions of $M(\Re)$. Equivalently, under the same hypothesis, any function continuous on $E$ (or just in $M(E)$ ) can be Carleman approximated on $E$ by functions which interpolate $f$ as assigned on a preassigned $f$-admissible interpolation subset of $E$.

\section{Proofs of basic theorems.}

LEMMA 1.1. A function $\varepsilon$ which is continuous on a closed set $E$ is an $\varepsilon_{E}$-function if and only if there exists $h \in A(\Re)_{O E}$ such that $\varepsilon(p) / h(p)$ is nonvanishing and continuous on $E$ after removal of removable singularities.

Proof. The set $Z$ of zeros on $E$ of an $\varepsilon_{E}$-function $\varepsilon$ is isolated. Hence, the Cousin Problem 2 is solvable ([12], p. 133); that is, by the generalized Weierstrass-factor theorem, there exists $h \in A(\Re)$ such that $h$ vanishes only at the zeros of $\varepsilon$ on $E$ and each of its zeros is of the same order as the corresponding zero of $\varepsilon$. Then $\varepsilon / h$ is nonvanishing and is continuous on $E$ except for removable singularities.

Conversely, if $\varepsilon / h$ is continuous and nonvanishing on $E$, then at a zero $q$ of $\varepsilon$ the function $h$ must have a zero, say of order $n_{q}$. Since $\lim _{p \rightarrow q}|\varepsilon(p) / h(p)|$ exists and is positive, there is a neighborhood $\mathscr{N}_{q}$ such that $\varepsilon(p) /(p-q)^{n_{q}}$ is continuous and bounded away from zero when $p \in\left(\mathscr{N}_{q} \cap E\right)$. Thus, $\varepsilon$ is an $\varepsilon_{E}$-function.

LEMMA 1.2. Suppose $f \in M(E)$, also that $\varepsilon$ is an f-admissible $\varepsilon_{E}$-function. Then there exists $h \in A(\Re)_{O E}$ such that (1) $\varepsilon / h$ is nonvanishing and continuous on $E$ and (2) $f / h \in M(E)$.

Proof. For $h$ defined as in Lemma 1.1, there is a neighborhood of each zero $q$ of $\varepsilon$ such that $f\left(p /(p-q)^{n q} \in M\left(E \cap \mathscr{N}_{q}\right)\right)$, whence a neighborhood such that $f / h \in M\left(E \cap \mathscr{N}_{q}\right)$. We conclude $f / h \in M(E)$.

Proof of Theorem 1. Suppose $\varepsilon$ is any $f$-admissible $\varepsilon_{E}$-function (alternately, an $f$-admissible $\varepsilon_{E, \infty}$-function). By Lemma 1.2 (alternately, the definition of an $f$-admissible $\varepsilon_{E, \infty}$-function), there exists $h \in A(\Re)_{O E}$, such that $|\varepsilon / h|$ is continuous and positive on $E$ (alternately, bounded away from zero on $E$ ) and $f / h \in M(E)$. By hypothesis, $f / h$ can be Carleman (uniformly) approximated on $E$ by $g \in M_{E \cup P}(\Re)$; that is, $\mid f(p) / h(p)$ $g(p)|\leq| \varepsilon(p) / h(p) \mid$, whence $|f(p)-g(p) h(p)| \leq|\varepsilon(p)|$. Since $g h \in$ 
$M_{E \cup p}(\Re)$, we conclude $f$ can be strongly- $C$ (str.-u.) approximated as required.

To prove the converse, let $\varepsilon=\varepsilon(p)$ be any function that is continuous and nonvanishing on $E$ (alternately, an arbitrary positive constant). Suppose $h \in A(\Re)_{O E}$ and that $f / h \in M(E)$. By Lemma 1.1 (or by definition) the function $\varepsilon h$ is an $\varepsilon_{E}$ (alternately, $\varepsilon_{E, \infty}$ )-function. By hypothesis, there exists $\theta \in M_{E \cup P}(\Re)$ such that $|f(p)-\theta(p)| \leq|\varepsilon(p) h(p)|$ on $E$, whence $|f(p) / h(p)-\theta(p) / h(p)| \leq|\varepsilon(p)|$, as required for Carleman (or uniform) approximation of $f / h$ on $E$, since $\theta / h \in M_{E \cup P}(\Re)$.

Lemma 2.1. Suppose $E$ is a closed set on $\Re$, where $\Re$ is an open Riemann surface, and that $P \subset \Re \backslash E$. If $f$ can be strongly-Carleman approximated on $E$ by functions of $M_{E \cup P}(\Re)$, then $f$ can be Carleman approximated on $E$ by functions of $M_{E \cup P}(\Re)$, which interpolate $f$ on $a$ preassigned f-admissible interpolation subset $Z$ of $E$.

Proof. By the generalized Weierstrass-factor theorem (that is, the solution of the Cousin Problem 2 ([12], p. 133)), there exists $h \in A(\Re)$ which has its zeros only at the points $q$ of $Z$, with each zero of the order $k_{q}$ of $f$ at that point.

Let $\delta=\delta(p)$ be an arbitrary function which is continuous and nonvanishing on $E$. Then for $\eta(p)=\min (|h(p)|,|\delta(p)|)$, the function $\eta$ is an $f$-admissible $\varepsilon_{E}$-function.

By the hypothesis that $f$ can be str.- C. approximated on $E$, there exists $g \in M_{E \cup P}(\Re)$ such that $|f(p)-g(p)| \leq|\eta(p)|$ when $p \in E$. Hence, $|f(p)-g(p)| \leq|\delta(p)|$ when $p \in E$, and at each point $q \in Z$ we have $g(q)$ equals $f(q)$. If $q \in Z$, there is $\mathscr{N}_{q}$ in which $\eta(p)=|h(p)|$, whence $|f(p)-g(p)| \leq|p-q|^{k_{q}}\left|h_{q}(p)\right|$, where $h_{q} \in A(\Re)$ and $h_{q}$ is nonvanishing in $\mathscr{N}_{q}$. Hence, $(f(p)-g(p)) /(p-q)^{k_{q}}$ is bounded and belongs to $A\left(\mathcal{N}_{q} \cap E\right)$. Thus, $g$ interpolates $f$ on $Z$.

In the case $\Re$ is the complex plane, the method of proof for the next lemma was used in [25].

Lemma 2.2. Suppose $E$ is a closed set on $\Re$ and that $P \subset \Re \backslash E$. Then an arbitrary function of $M(E)$ can be Carleman (alternately, uniformly) approximated on $E$ by functions of $M_{E \cup P}(\Re)$ if and only if an arbitrary function of $A(E)$ can be Carleman (alternately, uniformly) approximated on $E$ by functions of $M_{P}(\Re)$. An arbitrary function of $M(E)$ can be Carleman (alternately, uniformly) approximated on $E$ by functions of $M_{E}(\Re)$ if and only if an arbitrary function of $A(E)$ can be similarly approximated by functions of $A(\Re)$. 
Proof. The set $S$ of poles of $m \in M(E)$ is isolated. On $\Re$, which is a Stein manifold, the first Cousin Problem is solvable ([22], p. 259 and [12], p. 132). Hence, the generalized Mittag-Leffler Theorem implies there exists $h \in M_{S}(\Im i)$ such that, at each pole of $m, h$ has a pole of the same order with the saıne principal part.

Now, the function $m-h$ has removable singularities on $E$. Hence, by hypothesis, for an arbitrary positive continuous function $\varepsilon=|\varepsilon(p)|$ (alternately a positive constant $\varepsilon)$, there exists $g \in M_{P}(\Re)$ such that

$$
|(m(p)-h(p))-g(p)| \leq|\varepsilon(p)| \quad \text { on } E \text {. }
$$

Then

$$
|m(p)-(h(p)+g(p))| \leq|\varepsilon(p)| \text { on } E .
$$

We note that $h+g \in M_{E \cup P}(\Re)$. Furthermore, if $g \in A(\Re)$, then $(h+g) \in M_{S}(\Re)\left(\subset M_{E}(\Re)\right)$. Since the converse is trivial, this completes the proof.

Proof of Theorem 2. By Lemma 2.2, (a) implies (b). We next show that (b) implies (c). For arbitrary $f \in M(E)$ and $h \in A(\Re)_{O E}$ such that $f / h \in M(E)$, assumption (b) implies $f / h$ can be Carleman (or uniformly) approximated on $E$ by functions of $M_{E \cup P}(\Re)$. Then, by Theorem $1, f$ can be str.-C. (or str.-u.) approximated on $E$ by functions of $M_{E \cup P}(\Re)$. This completes the proof of (c).

For Carleman approximation, application of Lemma 2.1 yields (d). Since (a) is a special case of (d) or of (c), this completes the proof.

Proof of Corollary 2.3. Since $\left(\mathrm{h}_{2}\right)$ is just (f) of Scheinberg's Theorem (see [23]), it follows from (a) of that theorem, under the hypothesis that every function of $A(E)$ is locally approximable on $E$ by functions of $A(\Re)$, that every function of $A(E)$ can be uniformly approximated on $E$ by functions of $M_{P}(\Re)$. (See also Gauthier ([10], Theorem 1) for the case $P$ is not assigned.) Now, by (a) and (c) of Theorem 2 above, every function of $M(E)$ can be str.-u. approximated on $E$ by functions $M_{E \cup P}(\Re)$.

Proof of Theorem 3. By Scheinberg's Theorem ([23]: 1b), the function $f / h$ can be uniformly approximated on $E$ by functions of $M_{E \cup P}(\Re)$. It follows from Theorem 1 above that $f$ can be strongly uniformly approximated on $E$ by functions of $M_{E \cup P}(\Re)$.

Proof of Theorem 4. By a theorem of Gauthier (see [10], Theorem 4) combined with Lemma 2.2 above, any function meromorphic on $E$ which 
is locally approximable on $E$ by functions of $M(\Re)$ can be Carleman approximated on $E$ by functions of $M(\Re)$. Then, by (b) and (c) of Theorem 2 (Corollary 2.1) above, $f$ can be str.-C. approximated on $E$ by functions of $M(\Re)$. The proof is completed by applying (d) of Theorem 2 .

3. Some sufficient point set conditions for strong approximation. In this section a few examples are given illustrating the application of Carleman (or uniform) approximation theorems in order to obtain strong-Carleman (or strong-uniform) approximation for the same point sets. Work by Gauthier and Scheinberg gives relevant results, and also includes extensive references to related material. (See also a paper of H. J. Landau [14].)

The following corollary was previously proved by the author (see [26]) for the case $f \in A(E)$ with interpolation points only at points of tangency of the circles. Hence, this more general result is implied by Theorem 2 above.

Corollary 2.4. Let $\Re$ be the complex plane: $|z|<\infty$. Suppose $E=$ $\cup_{i=1}^{\infty} R_{i}$, where $R_{i}=\{z /|z-i| \leq 1 / 2\}$ and that $f \in M(E)$. Then $f$ can be Carleman approximated on $E$ by meromorphic functions whose only poles coincide with those of $f$ on $E$ and which interpolate $f$ on any preassigned $f$-admissible interpolation subset of $E$; equivalently, $f$ can be strongly Carleman approximated on $E$ by functions of $M_{E}(\Re)$.

The next corollary follows from Theorem 4 and the Weierstrass approximation theorem. (See also [26].)

COROLlaRY 4.1. A function which is continuous on the real line $R$ can be Carleman approximated on $R$ by an entire function which interpolates $f$ on any pre-assigned f-admissible interpolation subset of $R$.

We let $\Re^{*}$ denote the one point compactification of $\Re$; that is, $\Re^{*}=\Re \cup\{\infty\}$.

THEOREM 5. Suppose $E$ is closed and essentially of finite genus on an open Riemann surface $\Re$, and that $\Re^{*} \backslash E$ is connected and locally connected. Then every $f \in A(E)$ can be strongly uniformly approximated on $E$ by functions of $A(\Re)$ and every $g \in M(E)$ can be strongly-uniformly approximated on $E$ by functions of $M_{E}(\Re)$.

Proof. By a theorem of Scheinberg [22] (see also Gauthier [10], Theorem A.), arbitrary $f \in A(E)$ can be uniformly approximated on $E$ by 
functions of $A(\Re)$. Since (a) of Theorem 2 above holds with $P=\Re \backslash E$, (c) of that theorem yields the required conclusion.

A set $E$ in the complex plane $|z|<\infty$ is a $Q$-set if its components $E_{j}$ are compact and if no sequential limit point of $E$ belongs to $E$. (See [25], [26], [27].) A point $b$ that is a limit point of a set of points chosen one from each component $E_{j}$ of $E$ is a sequential limit point of $E$; that is, $b \in \varlimsup\left\{E_{j}\right\}$. (See [28].)

We let $B=\left\{\overline{\lim }\left\{E_{j}\right\}\right\}$. Then, for $\mathfrak{C}^{*}=\mathbb{} \cup\{\infty\}$, where $\mathbb{E}$ is the complex plane, the surface $\complement^{*} \backslash B$ is an open Riemann surface (actually, the union of open Riemann surfaces, in case $B$ separates the plane). We note that, relative to $\mathbb{C}^{*} \backslash B$, a $Q$-set is closed.

In the case $\Re=\mathbb{E} \backslash B$, where $\mathbb{E}$ is the complex plane $|z|<\infty$, the following theorem is given by Corollaries 2.1 and 2.2 in [27] for the case the zeros of $\varepsilon$ are interior points of $E$. (For the analogous uniform approximation result, see [4]; [5]; [15]; and [23].) The strong approximation result, for the case $\Re$ is an open Riemann surface, follows directly from Theorem 2 or Corollary 2.2 above, combined with Corollaries 3 and 4 as stated and reproved by Scheinberg in [23].

THEOREM 6. Suppose $E$ is a compact set on an open Riemann surface $\Re$ and that every bounded component of $\Re \backslash E$ contains a point of $P$. Then any $f \in H(E)$ can be strongly-uniformly approximated on $E$ by functions of $M_{P}(\Re)$. If no non-empty component of $\Re \backslash E$ is bounded, then any $f \in A(E)$ can be strongly-uniformly approximated on $E$ by functions of $A(\Re)$.

For a proof of Theorem 7 below, see [27], Corollary 2.3. Also, for a special connected unbounded closed set $E$ which does not separate the plane, it was shown, under the assumption that $\varepsilon=\varepsilon(z)$ does not vanish on $\partial E$, that $A(\Re)$ is strongly-dense in $A(E)$. (See [27], Corollary 3.3.)

THEOREM 7. Suppose $E$ is a $Q$-set on $\Re=\mathbb{C}^{*} \backslash B$. Let $P$ consist of one point from each bounded component of $\Re \backslash E$. Then arbitrary $f \in N(E)$ can be strongly-Carleman approximated on $E$, by functions meromorphic on $\Re$, with any poles on $E \cup P$.

\section{REFERENCES}

[1] N. U. Arakelyan, Uniform approximation on closed sets by entire functions [Russian], Akad. Nauk SSSR. Isvestia Ser. Mat., 28 (1964), 1187-1206.

[2] _ Approximation complexe et proriétés des fonctions analytiques, Actes, Congrès Intern. Math., 2 (1970), 595-600.

[3] H. Behnke and F. Sommer, Theorie der analytischen Funktionen einer complexen Veränderlichen, zweite veränderte Auglage, Springer-Verlag, 1962. 
[4] H. Behnke and K. Stein, Entwicklung analytischer Funktionen auf Riemannschen Flächen, Math. Ann., 120 (1949), 430-461.

[5] E. Bishop, Subalgebras of functions on a Riemann surface, Pacific J. Math., 8 (1958), 29-50.

[6] Leon Brown, P. M. Gauthier, and W. Seidel, Possibility of complex asymptotic approximation on closed sets, Math. Ann., 218 (1975), 1-8.

[7] T. Carleman, Sur un théorème de Weierstrass, Ark Mat. Astr. Fys., 20B, no. 4 (1927), $1-5$.

[8] P. M. Gauthier, Analytic approximation on closed subsets of open Riemann surfaces, Proc. conf. on constructive function theory, Blagoevgrad, Sofia (1977).

[9] , Tangential approximation on closed subsets of open Riemann surfaces, Proc. conf. on constructive function theory, Blagoevgrad Sofia.

[10] Meromorphic uniform approximation on closed subset of open Riemann surfaces, Approximation theory and functional analysis, J. B. Prolla (ed.), North-Holland publishing company, 1979.

[11] P. M. Gauthier and W. Hengartner, Uniform approximation on closed sets by functions analytic on a Riemann surface, Approximation Theory (Z. Ciesielski and J. Musielak, eds.) Reidel Holland (1975), 63-70.

[12] Lars Hörmander, An Introduction to Complex Analysis in Several Variables, D. Van Nostrand Company, Inc., 1966.

[13] Laura Ketchum-Kodama, Boundary measures of analytic differentials and uniform approximation on a Riemann surface, Pacific J. Math., 15 (1965), 1261-1277.

[14] H. J. Landau, On uniform approximation to continuous functions by rational functions with preassigned poles, Proc. Amer. Math. Soc., 5 (1954), 671-676.

[15] S. N. Mergelyan, On the representation of functions by series of polynomials on closed sets (Russian), Doklady Akademïa Nauk USSR, vol. 78, 405-408, Amer. Math. Soc. Translation No. 85.

[16] A. H. Nersesian, On the Carleman sets, (Russian), Izv. Akad. Nauk Arm. SSSR, 6 (1971), 465-471.

[17] On uniform and tangential approximation by meromorphic functions (Russian), Izv. Akad. Nauk Arm. SSSR, 7 (1972), 405-412.

[17a] Uniform approximation with simultaneous interpolation by analytic functions (Russian), Izv. Akad. Nauk Arm. SSSR, 15 (1980), 249-257.

[18] Alice Roth, Approximationseigenschaften und Strahlengrenzwerte unendlich vieler linearer Gleichungen, Comm. Math. Helv., 11 (1938), 77-125.

[19] Meromorphic approximationen, Comment. Math. Helv., 48 (1973), 151-176.

[20] Uniform and tangential approximation by meromorphic functions on closed sets, Canad. J. Math., 1 (1976), 104-111.

[21] S. Scheinberg, Uniform approximation by entire functions, J. d'Analyse Math., 29 (1976), 16-19.

[22] Uniform approximation by functions analytic on a Riemann surface, Ann. Math., 108 (1978), 257-298.

[23] Uniform approximation by meromorphic functions having prescribed poles, Math. Ann., 243 (1979), 83-93.

[24] Annette Sinclair, Generalization of Runge's theorem to approximation by analytic functions, abstract of a thesis, Univ. of Illinois (1949).

[25] , Generalization of Runge's theorem to approximation by analytic functions, Trans. Amer. Math. Soc., 72 (1952), 148-164.

[26] A A general solution for a class of approximation problems, Pacific J. Math., 8 (1958), 857-866.

[27],$|\varepsilon(z)|$-closeness of approximations, Pacific J. Math., 15 (1965), 1405-1413. 
[28] G. T. Whyburn, Analytic topology, Amer. Math. Soc. Colloq. Publ., vol. 28, Edwards Bros. Inc., Ann Arbor, Mich., 1948.

Received February 11, 1981 and in revised form December 14, 1983.

PuRdue UnIVERSITY

WEST LAFAYETTE, IN 47907 


\section{PACIFIC JOURNAL OF MATHEMATICS EDITORS}

DONALD BABBITT (Managing Editor)
University of California
Los Angeles, CA 90024
CHARLES R. DEPrIMA
California Institute of Technology
Pasadena, CA 91125
R. FINN
Stanford University
Stanford, CA 94305

\author{
HERMANN FLASCHKa \\ University of Arizona \\ Tucson, AZ 85721 \\ RAMESH A. GANGOLLI \\ University of Washington \\ Seattle, WA 98195 \\ ROBION KIRBY \\ University of California \\ Berkeley, CA 94720 \\ C. C. Moore \\ University of California \\ Berkeley, CA 94720
}

Hugo Rossi

University of Utah

Salt Lake City, UT 84112

H. SAMELSON
Stanford University
Stanford, CA 94305

HAROLD STARK

University of California, San Diego

La Jolla, CA 92093

\section{ASSOCIATE EDITORS}
R. ARens
E. F. BECKENBACH (1906-1982)
B. H. NeUmanN
F. WOLF
K. YOSHIDA

\begin{tabular}{ll}
\multicolumn{2}{c}{ SUPPORTING INSTITUTIONS } \\
UNIVERSITY OF ARIZONA & UNIVERSITY OF OREGON \\
UNIVERSITY OF BRITISH COLUMBIA & UNIVERSITY OF SOUTHERN CALIFORNIA \\
CALIFORNIA INSTITUTE OF TECHNOLOGY & STANFORD UNIVERSITY \\
UNIVERSITY OF CALIFORNIA & UNIVERSITY OF HAWAII \\
MONTANA STATE UNIVERSITY & UNIVERSITY OF TOKYO \\
UNIVERSITY OF NEVADA, RENO & UNIVERSITY OF UTAH \\
NEW MEXICO STATE UNIVERSITY & WASHINGTON STATE UNIVERSITY \\
OREGON STATE UNIVERSITY & UNIVERSITY OF WASHINGTON
\end{tabular}

The Supporting Institutions listed above contribute to the cost of publication of this Journal, but they are not owners or publishers and have no responsibility for its content or policies.

\footnotetext{
Mathematical papers intended for publication in the Pacıfic Journal of Mathematıcs should be in typed form or offset-reproduced (not dittoed), double spaced with large margins. Please do not use built up fractions in the text of the manuscript. However, you may use them in the displayed equations. Underline Greek letters in red, German in green, and script in blue. The first paragraph must be capable of being used separately as a synopsis of the entire paper. In particular it should contain no bibliographic references. Please propose a heading for the odd numbered pages of less than 35 characters. Manuscripts, in triplicate, may be sent to any one of the editors. Please classify according to the scheme of Math. Rev ews, Index to Vol. 39. Supply name and address of author to whom proofs should be sent. All other communications should be addressed to the managing editor, or Elaine Barth, University of California, Los Angeles, California 90024.

There are page-charges associated with articles appearing in the Pacific Journal of Mathematics. These charges are expected to be paid by the author's University, Government Agency or Company. If the author or authors do not have access to such Institutional support these charges are waived. Single authors will receive 50 free reprints; joint authors will receive a total of 100 free reprints. Additional copies may be obtained at cost in multiples of 50 .
}

The Pacific Journal of Mathematics is issued monthly as of January 1966. Regular subscription rate: $\$ 190.00$ a year (5 Vols., 10 issues). Special rate: $\$ 66.00$ a year to individual members of supporting institutions.

Subscriptions, orders for numbers issued in the last three calendar years, and changes of address should be sent to Pacific Journal of Mathematics, P.O. Box 969, Carmel Valley, CA 93924, U.S.A. Old back numbers obtainable from Kraus Periodicals Co., Route 100, Millwood, NY 10546.

The Pacific Journal of Mathematics at P.O. Box 969, Carmel Valley, CA 93924 (ISSN 0030-8730) publishes 5 volumes per year. Application to mail at Second-class postage rates is pending at Carmel Valley, California, and additional mailing offices. Postmaster: Send address changes to Pacific Journal of Mathematics, P.O. Box 969, Carmel Valley, CA 93924.

PUBLISHED BY PACIFIC JOURNAL OF MATHEMATICS, A NON-PROFIT CORPORATION Copyright $\odot 1985$ by Pacific Journal of Mathematics 


\section{Pacific Journal of Mathematics}

\section{Vol. 117, No. 2 \\ February, 1985}

Robert Walter Bagley, Ta-Sun Wu and J. S. Yang, On a class of topological groups more general than SIN groups ............... 209

Bruce Alan Barnes, Algebraic elements of a Banach algebra modulo an ideal ...............................................219

Howard D. Fegan and Peter Gilkey, Invariants of the heat equation .......233

Erica Flapan, Necessary and sufficient conditions for certain homology

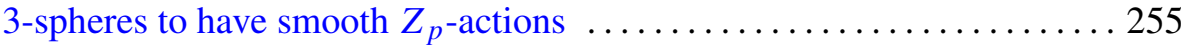

Philip R. Heath, Product formulae for Nielsen numbers of fibre maps . . . . 267

Derbiau Frank Hsu and A. Donald Keedwell, Generalized complete mappings, neofields, sequenceable groups and block designs. II ...... 291

Taqdir Husain, Orthogonal primitive idempotents and Banach algebras

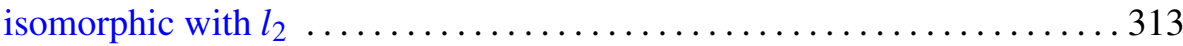

Gary M. Lieberman, Regularized distance and its applications . . . . . . . 329

William W. Menasco, Determining incompressibility of surfaces in alternating knot and link complements ............................ 353

Benjamin Muckenhoupt, Weighted reverse weak type inequalities for the Hardy-Littlewood maximal function $\ldots \ldots \ldots \ldots \ldots \ldots \ldots \ldots \ldots \ldots \ldots \ldots$

John Dacey O'Neill, Direct summands of direct products of slender

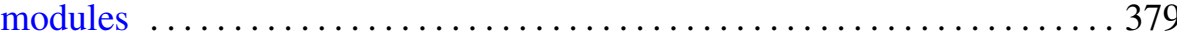

Andreas Rapp, Elimination of Malitz quantifiers in stable theories 387

Francisco José Ruiz, A unified approach to Carleson measures and $A_{p}$ weights

Hanamatagouda Pandappa Sankappanavar, Heyting algebras with dual

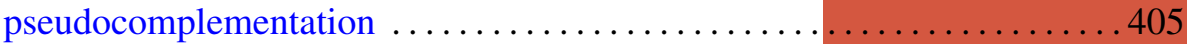

Annette Sinclair, Strong Carleman and strong uniform approximation . . . . 417

Masaaki Suzuki, The generalized Schwarz lemma for the Bergman metric

Brian Thorpe and Ludwig Tomm, Universal approximation by regular

weighted means 